\section{Talking to Machines}

Understanding Natural Language. By Terry Winograd. Pp. vi+191. (Academic: New York and London; Edinburgh University: Edinburgh, May 1972.) $\$ 8.95$.

ASKED to say what artificial intelligence has to show for its first fifteen years, any practitioner is bound to say "Terry Winograd's program". Anyone who has seen the filmed dialogue between program and user - part of which is reproduced in this book-cannot fail to be impressed by the naturalness with which the computer program carries out commands, and provides informative answers both about the current state of its world, and recent events involving the program's manipulation of that world.

The world of the program is a large collection of formulae or "assertions" which can be operated upon by procedures. Collectively these procedures and assertions make up a data base which describes in a minimal fashion a table top with coloured blocks of various shapes and sizes on it, and a variety of actions and relations in that world. Each object is located on a three dimensional coordinate grid: thus (\# AT: B3 (400 00 )) asserts that a block whose "internal" name is "B3" is located at $x=400, y=0, z=0$. Assertions of the same general form are a prominent feature of the procedures which represent actions like GET RIDOF, PUTON, GRASP, MAKESPACE and so on, in such a way that a request to "put $X$ on $Y$ " can, after checking that (\#ON X Y) is not already asserted (that is $\mathrm{X}$ is not already on $\mathrm{Y}$ ), cause a search over the procedures to locate one whose asserted goal is (\#PUTON X Y). Activation of this procedure creates new assertional requirements which may be satisfied in turn by other procedures and so on.

On this computational deep structure an account of natural language and its relation to intelligence and cognition is constructed which is at once more explicit, more detailed and fundamentally more plausible than anything else on the book shelves. It is more plausible because, at last, there is a honest attempt to grapple with meaning and reference regarded as a translation of the user's sentences into computational forms which have some chance of coping with behaviour in a real world. The requirements of this translation motivate a detailed linguistic theory which uses a "systemic" grammar to determine the form of the complex syntactic structures which are assigned in understanding the sentence. This departure from the more fashionable transformational grammar of Chomsky is strongly influenced by a concern to reflect the speaker/hearer's knowledge of the language in the process of comprehension, not merely in the syntactic structures themselves. Winograd pursues this theme further by exploiting a version of the semantic theory of Katz and Fodor to smooth the path of efficient understanding. In his hands that theory is a heuristic technique which both reflects and merges into the speaker/hearer's knowledge of the world.

In the early 1960s Bar-Hillel "showed" the impossibility-in-principle of mechanical translation of natural languages by drawing attention to the way in which a knowledge of real world objects and relationships is involved in language use. He illustrated the point by drawing attention to the ambiguity of the word "pen" in the sentences "the pen is in the box" and "the box is in the pen". He concluded that such a disambiguation was impossible because one could not provide a machine with such knowledge of the real world or the capacities to use it. The requisite inferences here, having to do with relative sizes and locations, are precisely those captured by the computations performed in Winograd's blocks world: it is the pervasive influence of such varieties of inference in the understanding of utterances that is a central thesis of Winograd's work.

Having made much of the centrality of meaning and reference it may disappoint the reader to find that a third of the account of the system is devoted to a grammar of English and even more surprising that the fundamental driving force is provided by the parsing of the utterance. The perceptual and manipulative actions in the blocks world, generated by the understanding of the user's utterance, are in a strict computational sense merely a side effect of a successful parse: an ideologically unsound but pragmatically successful tour de force. Of deeper concern to those who believe that utterances are much messier than the linguist appears to believe, is that despite overt deployment of meaning and reference it is still necessary that utterances be grammatically well formed and words correctly spelled.

These points should not be thought of as "defects" so much as problems whose solution will require a basic restructuring of the processes captured in the program. Winograd's achievement is to show how knowledge of the language and of the world can (and must) be expressed in the process of understanding just as much as in the structures (syntactic and semantic) upon which that process operates. It is this which distinguishes Winograd's work from the contemporary linguistic orthodoxy ushered in by Chomsky's Syntactic Structures and makes it required reading for any self respecting student of human nature.

The texts of the two editions of this book are identical save for the addition in the Edinburgh University Press copy of an index and a brief preface by the author.

MAX Clowes

\section{Enumeration}

Development of Modern Mathematics. By J. M. Dubbey. Pp. $7+145$. (Butterworth: London, November, 1970.) 50s boards; 28s paper.

IN recent years the history of mathematics has come to assume an importance much greater than at any time in the past. It is now a fashionable subject, both in its sociological and cultural aspects, and is becoming of increasing interest as a possible component in the curriculum at schools and colleges. But it is more than this. It is important in mathematics as such, both because of the tremendous developments of the past two centuries or more and also because of the great emphasis in contemporary mathematics on concepts and their relationships. Clearly in such ani emphasis there is a rightful place for a study of the historical development of the concepts.

In relation to these developments the appearance of the book under review is most timely. There is, in fact, a great dearth of literature on the history of mathematics, apart from a number of classical texts on Greek mathematics, and a noteworthy feature of this book is that more than two-thirds of it is given to mathematics as it has developed since the time of Newton. Particularly important are the chapters on eighteenth century mathematics and on the origins of modern mathematics. The author bravely attempts an introductory survey of twentieth century mathematics and it is understandable that he "trembles at the prospects". Under pure mathematics he has written a good, but brief, survey of ideas in analysis and in analytical topology, but a notable absence is any reference to the widespread developments in abstract algebra during the present century. As for applied mathematics there are brief references to computers and techniques, but a noticeable omission of any reference to some of the main branches of the subject, for example, fluid mechanics and electromagnetism. Having said this, it is only fair to say also that the book will give the reader a very good first impression of some of the main mathematical developments over recent decades. It should prove especially useful on undergraduate courses in the history of mathematics, provided the students already have a reasonable background in the mathematics whose history they are studying.

L. S. GODDARD 